\title{
Safety of Thrombolysis in a Patient with an Intracranial Dural Arteriovenous Fistula
}

\author{
Ana João Pissarra ${ }^{1}$, Mariana Malheiro ${ }^{1}$, Carolina Gouveia² Patrícia Vicente $^{2}$, Leonor Matos ${ }^{1}$ \\ ${ }^{1}$ Medical Oncology Department, Hospital São Francisco Xavier, Lisbon, Portugal \\ ${ }^{2}$ Internal Medicine Department, Hospital São Francisco Xavier, Lisbon, Portugal
}

Received: $14 / 12 / 2018$

Accepted: $24 / 12 / 2018$

Published: 14/01/2019

How to cite this article: Pissarra AJ, Malheiro M, Gouveia C, Vicente P, Matos L. Safety of thrombolysis in a patient with an intracranial dural anteriovenous fistula. EJCRIM 2019;6: doi:10.12890/2019_001019.

Conflicts of Interests: The Authors declare that there are no competing interests.

This article is licensed under a Commons Attribution Non-Commercial 4.0 License

Acknowledgements: The authors would like to thank Dr Vitor Batalha for the support provided in this clinical case.

\section{ABSTRACT}

Intracranial dural arteriovenous fistula is an abnormal connection between an artery and a vein that has an increased risk of bleeding. This case report presents a 53-year-old man diagnosed with a dural arteriovenous malformation fistula in occipital topography, lacking therapeutic indication because of an extension. He was admitted to an intensive care unit due to a high-risk pulmonary thromboembolism with indication for thrombolysis. Taking into account the hemorrhagic risk associated with arteriovenous malformation, the authors discuss the therapeutic options and the inherent risks.

\section{LEARNING POINTS}

- Intracranial dural arteriovenous fistulas are pathologic shunts between dural arteries and veins that have an inherent risk of intracranial hemorrhage.

- Systemic thrombolytic agents are a therapeutic option for high-risk pulmonary thromboembolism. Their potential benefits outweigh the risk of life-threatening bleeding; however, careful patient risk stratification should be performed and other options, such as surgical embolectomy or percutaneous catheter-directed treatment, should be considered if available.

- Multidisciplinarity is the key to better therapeutic decisions and the patient's opinion should always be taken into account.

\section{KEYWORDS}

Pulmonary thromboembolism, thrombolysis, arteriovenous malformation

\section{CASE PRESENTATION}

We present the case of a 53-year-old man with no relevant personal or familial history and on no regular medication. In 2011, he presented with pulsatile tinnitus, episodic dizziness and headache, without other neurological signs or symptoms. At physical examination, an exuberant bilateral carotid bruit was audible; continuous, more intense on the right side with retroauricular and occipital bilateral irradiation. No neurological signs were noticed. A computed tomography (CT) angiography of the brain was performed and revealed an arteriovenous malformation (AVM). To better characterize it, magnetic resonance angiography (MRA) was performed and showed multiple irregular vessels in right occipital topography, involving the jugular vein in right tentorial topography (Fig. 1). At this time, the diagnosis of AVM with dural fistulas was established (Fig. 2). The case was discussed in a multidisciplinary meeting with the neurosurgical team and, despite the absence of comorbidities, due to extension of the AVM, surgical correction or catheter embolization was not an option. The patient was subsequently monitored with periodic MRA (Fig. 3). 

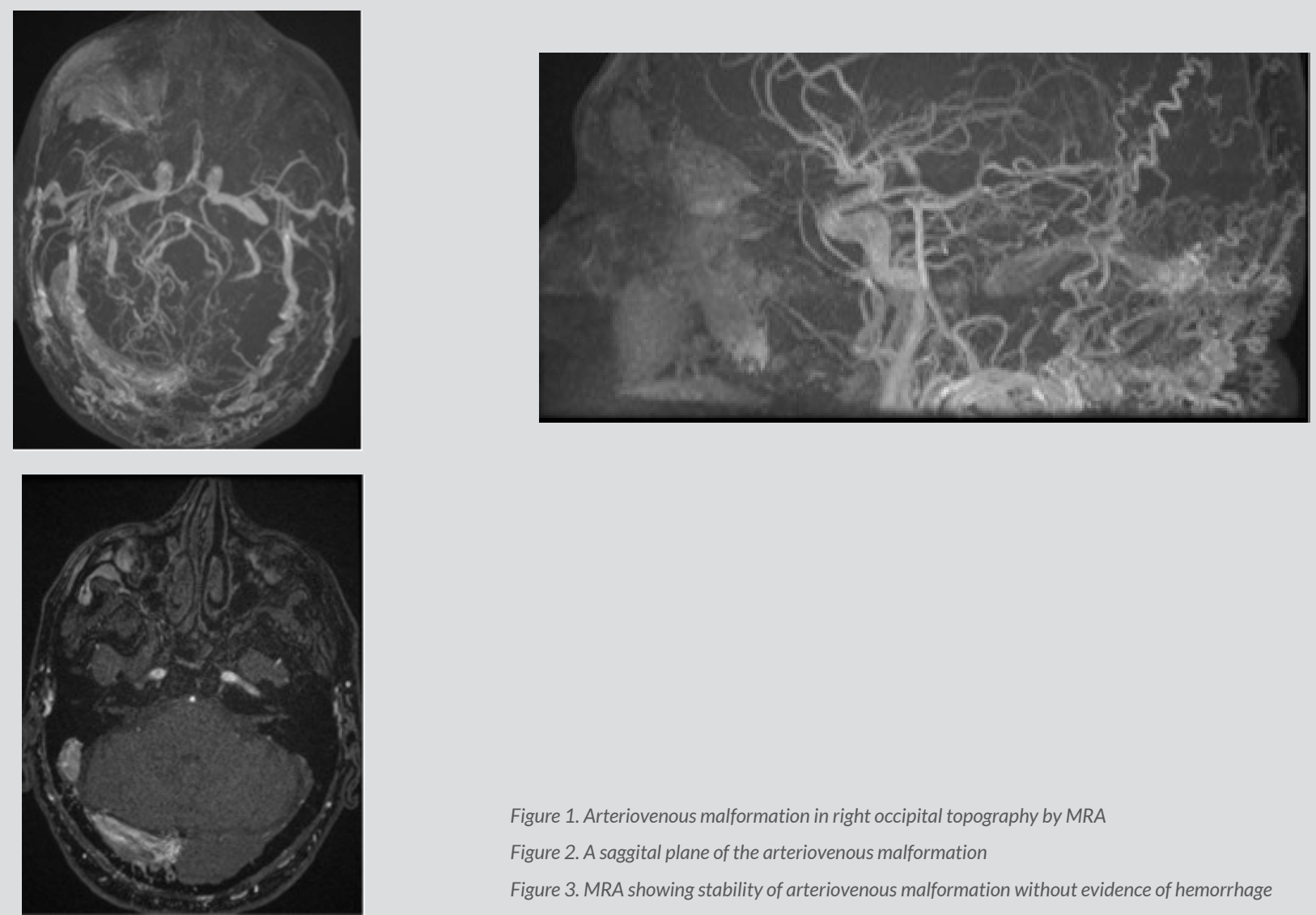

Figure 1. Arteriovenous malformation in right occipital topography by MRA

Figure 2. A saggital plane of the arteriovenous malformation

Figure 3. MRA showing stability of arteriovenous malformation without evidence of hemorrhage

Seven years later, in 2018, the patient attended the emergency department with retrosternal chest pain with a squeezing sensation, without irradiation, palpitations or dyspnea triggered on mild exertion. A previous episode of pain in his left leg was recorded.

At admission, he was hemodynamically unstable (mean arterial pressure 58-87 mmHg), with tachycardia (heart rate 120-140 bpm), bilateral carotid bruit, with retroauricular and occipital irradiation. From the complementary tests performed, the following were noted: an electrocardiogram showing a $\mathrm{S}_{1} \mathrm{Q}_{3} \mathrm{~T}_{3}$ pattern; gasometry with respiratory alkalosis, hypoxemia and hypocapnia; and elevated D-dimer and myocardial necrosis biomarkers. Thoracic CT angiography revealed an extensive bilateral pulmonary thromboembolism (PE), while a Doppler ultrasound of the lower extremities identified a deep venous thrombosis in the left popliteal vein. The echocardiogram had right ventricular dysfunction. The Pulmonary Embolism Severity Index (PESI) at this time was 154 points (very high risk group - with 30-day mortality 10-24.5\%). Taking into account the high-risk PE, thrombolysis was indicated. It was discussed with the neurosurgical team and having assessed the risk/benefit, despite the AVM, it was decided to opt for thrombolysis as a life-saving measure. The patient underwent thrombolytic therapy with alteplase $100 \mathrm{mg}$ IV infusion, with improvement of symptoms and absence of hemorrhagic complications. At 6-month follow-up, the patient reported no symptoms, with excellent functional status and MRA showed no alteration in AVM.

\section{DISCUSSION}

PE is a common clinical entity and the third most common cause of cardiovascular mortality. Hemodynamically unstable patients with shock or hypotension should immediately be identified as high-risk patients and are candidates for primary pharmacological or surgical/ interventional reperfusion therapy ${ }^{[1]}$. In the reported case, the PE-related risk and the patient's clinical status were indications for thrombolysis. Thrombolytic agents convert native plasminogen to plasmin, which hydrolyzes the fibrin, resulting in clot lysis. It improves arteriovenous oxygenation, pulmonary artery pressure and reperfusion, relieving symptoms, and reducing mortality ${ }^{[2]}$. This therapy has an associated risk of hemorrhage and the benefits may not outweigh the risk of bleeding, so it is important to consider the patient's bleeding potential before administering thrombolytic agents. There are no validated tools for predicting this risk, but some scores, like PESI, can help to identify patients with a high risk of mortality who may benefit from thrombolytic therapy. There are also some clinical risk factors that contraindicate thrombolysis, and one of the absolute contraindications is structural intracranial disease, as dural arteriovenous fistulas $(\mathrm{DAVF})^{[2,3]}$. 
DAVF are pathologic shunts between dural arteries and venous sinuses, meningeal veins, or cortical veins ${ }^{[4]}$. The majority present in the fifth/sixth decades and symptoms are related to lesion location and pattern of venous drainage. Pulsatile tinnitus is a common symptom that results from increased blood flow through the dural venous sinuses. Endovascular approaches have become the mainstay of therapy, but the optimal approach for each case should involve discussions among a multidisciplinary team ${ }^{[4,5]}$. Severe presentations include intracranial hemorrhage and the risk of bleeding is high ${ }^{[5]}$. Some studies conclude that hemorrhage from DAVF is often associated with cortical venous drainage, male predominance and tentorial type ${ }^{[6]}$.

Thrombolytic treatment carries a risk of major bleeding, including intracranial hemorrhage, and the reported data indicate intracranial bleeding rates between $1.9 \%$ and $2.2 \%$. Increasing age and the presence of comorbidities have been associated with a higher risk of bleeding complication ${ }^{[1]}$. Despite the absolute contraindications for thrombolysis, the evaluation must be individualized and most contraindications should be considered relative to patients with life-threatening, high-risk PE. In patients with absolute contraindications to thrombolysis, other approaches, such as surgical embolectomy or percutaneous catheter-directed treatment, should be evaluated.

In this clinical case, due to AVM, the risk of intracranial hemorrhage was higher and could be precipitated by the use of thrombolytic agents. The patient was discussed in a multidisciplinary team with the neurosurgical team and in assessing the risk/benefit, it was decided to perform thrombolysis with alteplase as a life-saving measure, because no other options were available.

There are some data that compare the bleeding rates after the administration of alteplase in patients who received heparin only and in patients who received alteplase plus heparin, and the rates of bleeding in the two groups did not differ significantly ${ }^{[7]}$.

In summary, systemic primary reperfusion treatment, particularly systemic thrombolysis, is the treatment of choice for patients with highrisk PE. In patients with contraindications to thrombolysis, surgical embolectomy or percutaneous catheter-directed treatment should be considered, if appropriate resource and expertise with these methods are available. If absolute contraindicated risk factors exist and no other techniques are available, thrombolysis should be performed because its potential benefits outweigh the risk of a life-threatening bleed. Treatment decisions should be made by a multidisciplinary team, ideally including a thoracic surgeon or interventional cardiologist. This case report highlights a dilemma in daily clinical practice in which it is necessary to make a decision between the scientific evidence and the patient's need. It reinforces the importance of a case-by-case decision within a multidisciplinary team.

\section{REFERENCES}

1. Konstantinides SV, Torbicki A, Agnelli G, Danchin N, Fitzmaurice D, Galié N et al. 2014 ESC Guidelines on the diagnosis and management of acute pulmonary embolism. Eur Heart J 2014;35:3033-3080.

2. Martin C, Sobolewski K, Bridgeman P, Boutsikaris D. Systemic thrombolysis for pulmonary embolism: a review. Pharm Ther 2016;41:770-775.

3. Smithburger PL, Campbell S, Kane-Gill SL. Alteplase treatment of acute pulmonary embolism in the intensive care unit. Crit Care Nurse 2013; 33:17-27.

4. Gandhi D, Chen J, Pearl M, Huang J, Gemmete JJ, Kahturia S. Intracranial dural arteriovenous fistulas: classification, imaging findings, and treatment. AJNR Am J Neuroradiol 2012;33:1007-1013.

5. Söderman M, Pavic L, Edner G, Holmin S, Andersson T. Natural history of dural arteriovenous shunts. Stroke 2008;39:1735-1739.

6. Daniels DJ, Vellimana AK, Zipfel GJ, Lanzino G. Intracranial hemorrhage from dural arteriovenous fistulas: clinical features and outcome. Neurosug Focus 2013;34:E15.

7. Konstantinides S, Tiede N, Geibel A, Olschewski M, Just H, Kasper W. Comparison of alteplase versus heparin for resolution of major pulmonary embolism. Am J Cardiol 1998;82:966-970. 